\title{
Territoires enclavées et opportunités de marché : analyse des performances logistiques des corridors de transport en Afrique subsaharienne
}

\author{
Yann Alix, $\mathrm{Ph} . \mathrm{D}$ \\ Fondation SEFACIL - Le Havre \\ Jean-François Pelletier, Doctorant, Université Paris.Est \\ Analyste, Innovation maritime - Rimouski
}

\section{TERRITOIRES ENCLAVÉS, DÉVELOPPEMENT ÉCONOMIQUE ET PERFORMANCE LOGISTIQUE}

Les experts de la Banque Mondiale rappellent que les coûts d'importation des produits internationaux à destination des marchés enclavés peuvent atteindre 15 à $20 \%$ dans les zones enclavés africaines contre moins de $5 \%$ dans le contexte des pays industrialisés.

Un élément incompressible pour qualifier l'handicap physique des territoires enclavés relève de la distance géographique séparant les marchés intérieurs des portes d'entrées/sorties portuaire ${ }^{1}$. La corrélation entre cette distance géographique et les surcoûts de pré et postacheminements terrestres a été largement documenté, notamment dans le contexte des pays en voie de développement ${ }^{2}$. Les experts de la Banque Mondiale ${ }^{3}$ rappellent que les coûts d'importation des produits internationaux à destination des marchés enclavés peuvent atteindre 15 à $20 \%$ dans les zones enclavés africaines contre moins de $5 \%$ dans le contexte des pays industrialisés.

La réciproque est vraie dans le sens des exportations où la compétitivité internationale des productions des pays enclavés se trouve tout autant imputée par ces surcoûts liés en grande partie au facteur transport. Pour une insertion compétitive des zones enclavées africaines dans les circuits internationaux des échanges, il faut s'assurer d'une logistique de transport alignée avec les besoins de la demande mondiale. Il est intéressant de reprendre les propos du Président de la Banque Africaine de Développement : « la baisse des exportations et la variabilité du prix des matières premières projettent le PIB africain à $2 \%$ de croissance en 2009 [...] qu'une économie chinoise au-dessus de $10 \%$ place la totalité des économies africaines dans une perspective à $+6 \%$ de croissance du PIB continental! » ${ }^{4}$.

\section{Pour une insertion compétitive des zones enclavées africaines dans les circuits internationaux des échanges, il faut s'assurer d'une logistique de transport alignée avec les besoins de la demande mondiale. [...] la baisse des exportations et la variabilité du prix des matières premières projettent le PIB africain à $2 \%$ de croissance en 2009 [...] qu'une économie chinoise au-dessus de $10 \%$ place la totalité des économies africaines dans une perspective à $+6 \%$ de croissance du PIB continental!}

Outre la géographie, les pays enclavés subissent une combinaison interactive de facteurs très variés. La figure 1 reprend les principales catégories de facteurs qui contraignent le positionnement pérenne des productions enclavées sur les marchés mondiaux de consommation et surtaxent les produits internationaux consommés dans les pays sans littoral. 
Figure 1 : La qualification de l'enclavement d'un territoire : une somme complexe de contraintes en interaction dynamique

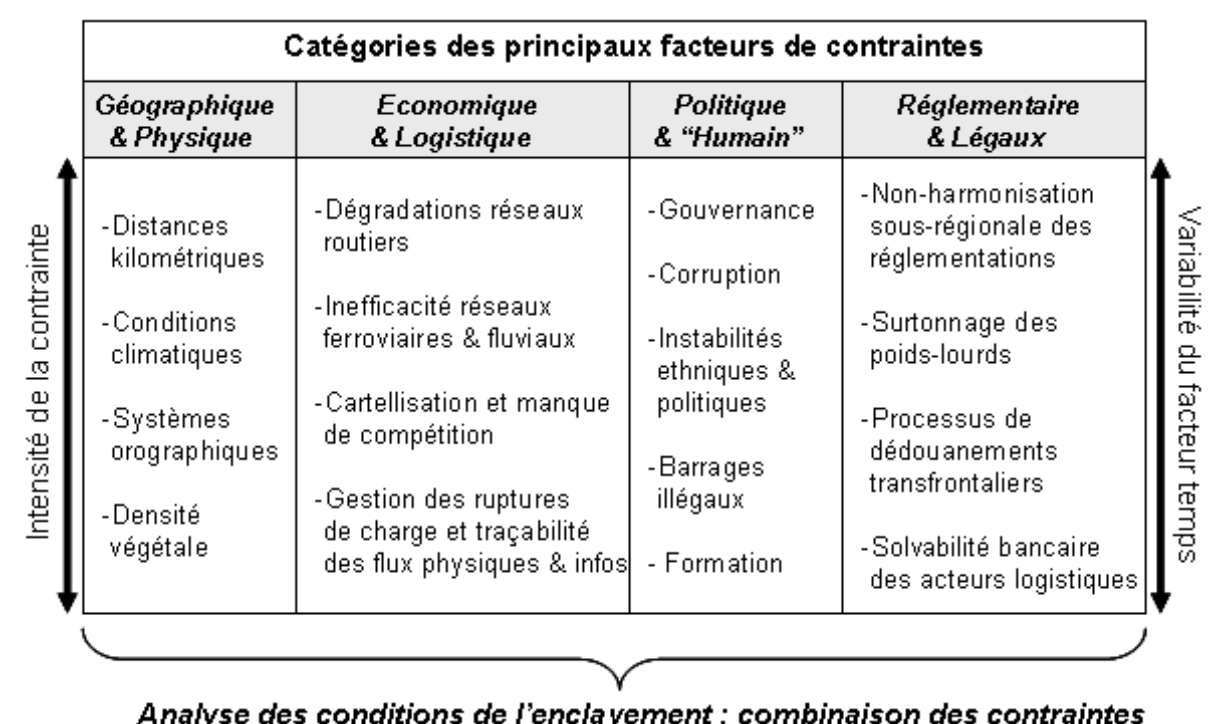

Seuls les éléments liés à la géographie physique (distance) et climatique (saison des pluies, tempêtes tropicales ou de sable, etc.) demeurent difficilement modifiables par une action anthropiquea. Même les obstacles orographiques trouvent des solutions par le génie civil dans la construction d'ouvrages d'arts comme les tunnels ou les ponts.

La classification par ordre d'impact ou d'importance d'une catégorie de facteurs vis-àvis d'une autre relève de l'analyse au cas par cas. C'est pourquoi les indices d'intensité et de temporalité des contraintes se placent dans une logique transversale signifiant que chaque pays ou zones enclavées présentent leurs propres combinaisons, intensités et temporalités de contraintes. Toutefois, de ces principales catégories, il est intéressant de noter que seuls les éléments liés à la géographie physique (distance) et climatique (saison des pluies, tempêtes tropicales ou de sable, etc.) demeurent difficilement modifiables par une action anthropiquea. Même les obstacles orographiques trouvent des solutions par le génie civil dans la construction d'ouvrages d'arts comme les tunnels ou les ponts. Aussi, force est de constater que la plupart des contraintes de l'enclavement relève de facteurs humains qui pourraient, selon l'intensité et le temps des actions correctives, se trouver en partie résolue. Cette assertion reste d'autant plus intéressante si l'on se concentre sur les modalités des acheminements terrestres et l'envergure logistique des circuits de transport, en particulier dans les zones enclavées de l'Afrique subsaharienne.

Que nous évoquions la qualité des réseaux routiers, la désuétude des équipements ou encore l'inefficacité des réseaux de transmission des données informatiques, les pays enclavés subissent directement les conséquences d'un triptyque négatif : faibles investissements $=$ prix élevés $=$ médiocrité des services.

Il en résulte que les coûts globaux de transport demeurent anormalement élevés s'ils sont mis en perspective de la qualité générale des services rendus. Le directeur Général du Conseil Burkinabé des Chargeurs, rappelait que le coût de transport et du transit atteignait $15 \%$ du prix de revient total CAF Abidjan pour une tête de bétail, $30 \%$ pour un kilo de viande et même $35 \%$ pour un kilo d'arachide 5 . Selon la Banque Mondiale, les surcoûts terrestres de transport pour atteindre les marchés enclavés tchadiens atteignent de telles proportions que même le secteur aérien présente de meilleurs coûts totaux 
rapportés au transport d'une tonne sur un kilomètre $^{6}$. Dans des situations de crise humanitaire comme celle du Darfour, l'ampleur des coûts logistique et de transport est telle que les valeurs des produits alimentaires importés demeurent inférieures au total des coûts terrestres pour supporter leur acheminement.

En outre, les prix payés par les principaux chargeurs des zones enclavées s'avèrent d'autant plus impactant que les trafics concernent essentiellement des denrées agroalimentaires qui fluctuent au gré des cours mondiaux et restent très sensibles à la fiabilité d'un temps de transit terrestre rarement garanti. En moyenne, il faut compter 26 jours de transit entre le port de Cotonou et Niamey, 38 jours entre Lagos et Abéché ou encore 45 jours entre Douala et $\mathrm{N}^{\prime}$ Djamena ${ }^{7}$. Les prix et pratiques de transport pénalisent doublement l'émergence d'un secteur manufacturier qui reste d'autant plus handicapé à l'exportation du fait qu'il doive importer une grande partie des intrants ${ }^{8,9}$. Enfin, des pratiques que certains qualifient «d'anormales» ont même mené à la création d'un Observatoire des pratiques anormales en 2006 à l'initiative des associations de chargeurs des pays enclavés de l'Ouest de l'Afrique ${ }^{10}$. Le nombre d'arrêts obligatoires, les sommes dépensées et les temps perdus sont recensés afin de quantifier et qualifier les taxes directes/indirectes ponctionnées par une kyrielle d'acteurs des économies formelle et informelle. La surtaxe du Bordereau de Suivi de Cargaison togolais sur les marchandises en transit par le port de Lomé ou les contrôles inopinés qui peuvent atteindre selon les tronçons routiers ouest-africains 5 arrêts au 100 kilomètres en restent des exemples probants.

\section{Dans des situations de crise humanitaire comme celle du Darfour, l'ampleur des coûts logistique et de transport est telle que les valeurs des produits alimentaires importés demeurent inférieures au total des coûts terrestres pour supporter leur acheminement.}

C'est dans ce contexte général que le programme décennal ALMATY a été mis en place en 2003 sous l'impulsion de l'ONU et des ministres des pays enclavés et en transition afin de soutenir des actions directes sur: Transit Policy \& Regulatory Frameworks; Infrastructure Development; Trade \& Transport Facilitation; Development Assistance; Implementation and Review.

Il reste une problématique à résoudre dans le suivi et l'accompagnement de ces programmations ambitieuses : la collecte fiable et régulière de données et indicateurs reliés à la performance des transports et des circuits logistiques. Le questionnement est double. D'une part, quels données/indicateurs et quelle uniformisation des informations sur des ensembles géographiques régionaux cohérents. D'autre part, quelles méthodologies appliquées afin de transformer des données primaires en résultats logistiques sur la performance des services de transport depuis un port donné jusqu'à un marché enclavé particulier. C'est pour répondre à ces deux questions méthodologiques que la partie suivante expose les éléments fondamentaux à retenir pour cadrer une analyse logistique sur la desserte de marchés enclavés situés dans la partie septentrionale de l'Afrique subsaharienne.

\section{PRÉSENTATION GÉNÉRALE ET JUSTIFICATION DE LA ZONE ET DES CORRIDORS LOGISTIQUES À L'ÉTUDE}

En premier lieu, il convient de caractériser le potentiel de trafics issus des marchés enclavés retenus pour l'étude de performance logistique. Deux régions distinctes peuvent être identifiées selon des prérogatives naturelles et anthropiques: les régions économiques, administratives et démographiques des deux capitales intérieures (Niamey au Niger et N'Djamena au
Tchad) et les régions d'Agadès au Niger et d'Abéché au Tchad.

D'abord, le Niger et le Tchad sont parmi les plus pauvres de la planète avec des indicateurs de développement humain qui classent, en 2009, le Niger au dernier rang et le Tchad $175^{\mathrm{e}}$ sur un ensemble de 182 pays recensés. Néanmoins, un 
total potentiel d'environ 670000 et 520000 tonnes métriques internationales ont été estimées pour le Niger et le Tchad à partir des données officielles de commerce international des Nations-Unies. La quasi-totalité de ces potentiels trafics se situe dans le sens des importations avec environ $60 \%$ du total comprenant divers produits agroalimentaires afin de nourrir des populations de moins en moins rurales et de plus en plus regroupées sur les centres urbains des deux capitales politiques et économiques.

Ensuite, la région d'Agadès au Niger représente un potentiel de trafic d'exportation avec l'importance des gisements d'uranium alors que la région d'Abéché au Tchad concentre les foyers de populations déplacées par les troubles politiques et ethniques du Soudan et de l'est du Tchad. Les trafics estimés oscillent pour l'uranium nigérien à 3000 tonnes métriques uniquement dans le sens des exportations alors que 70000 tonnes métriques annuelles de trafics alimentaires sont considérées par les agences internationales à destination de la zone d'Abéché.

À l'heure actuelle, ces quatre aires de marché potentiel se caractérisent par un enclavement structurel particulièrement handicapant pour leur développement économique. Aucune combinaison logistique multimodale s'avère efficiente hormis dans le contexte exceptionnel des acheminements aériens ou militaires et dans l'utilisation très sporadique du tronçon ferroviaire reliant le port de Cotonou au nord du Bénin ou le lien ferroviaire camerounais vers Ngaoundéré. Considérant ces éléments structurels, quatre corridors de transport sont appréhendés dans les pré et post acheminements des imports/exports internationaux en provenance ou à destination des quatre aires de marché enclavé (Figure 2).

Figure 2 : Les 4 corridors et la zone internationale enclavée à l'étude

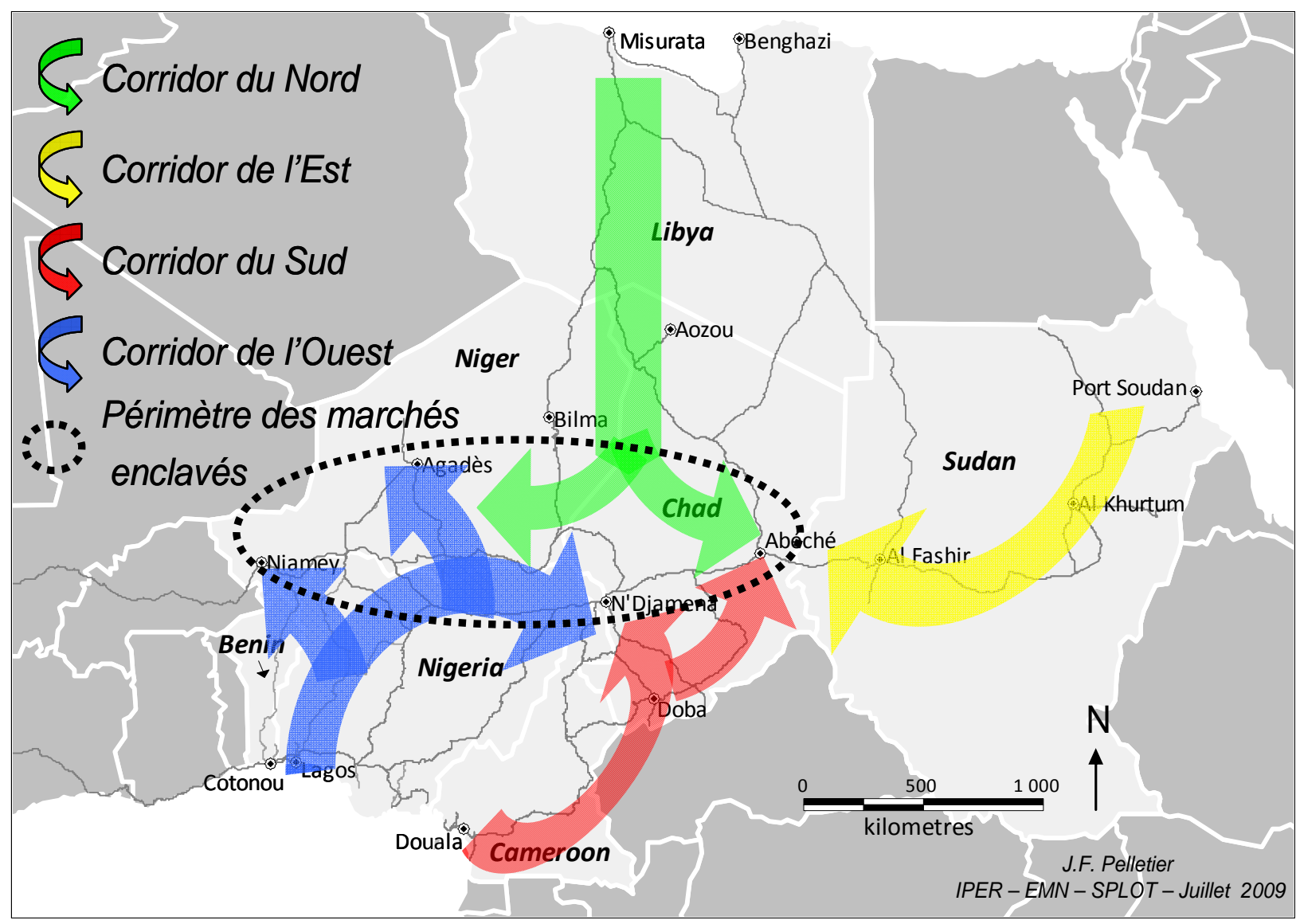


Le corridor de Misurata qui demeure l'option la moins usitée mais peut-être la plus prometteuse si l'on prend sérieusement en compte les ambitions du pouvoir libyen qui souhaite utiliser la manne pétrolière pour construire le premier tronçon de son axe routier panafricain ${ }^{11}$. Les accès facilités depuis la zone franche de Misurata positionne l'option du nord comme une alternative aux autres corridors actuellement en compétition malgré une situation géographique excentrée vis-à-vis des aires de marché considérées. En outre, les opportunités de consolidation de trafics sur le parcours restent très faibles du fait de l'absence de foyers importants de populations dans le sud de la Libye et le nord $\mathrm{du}$ Niger, terre particulièrement hostile avec notamment de nombreux champs de mines et des zones tribales de non-droits.

\section{Des estimations de la part de plusieurs}

ONG évoquent des taux de détournement de 65 à $90 \%$ des tonnages totaux transitant par Port Soudan et l'axe routier soudanais.

Le corridor de l'est avec le port de Port Soudan reste à l'heure d'aujourd'hui une option intéressante pour la desserte directe d'Abéché mais demeure particulièrement éloignée pour N'Djamena et surtout Agadès/Niamey. Le souci majeur de cette option logistique reste la corruption et le détournement d'une très grande partie des produits en transit vers les zones enclavées tchadiennes. Des estimations de la part de plusieurs ONG évoquent des taux de détournement de 65 à $90 \%$ des tonnages totaux transitant par Port Soudan et l'axe routier soudanais. Les troubles ethniques, les multiples conflits régionaux et la revente au marché noir des produits détournés constituent les principales contraintes « logistiques » de cette option sousrégionale, sans évoquer les soucis douaniers, réglementaires et fiscaux rencontrés directement avec l'administration soudanaise du port et au passage frontalier avec le Tchad.

Les corridors de Cotonou à l'Ouest et Douala au Sud demeurent les options les plus fiables si on les compare aux autres, mais des contraintes spécifiques demeurent handicapantes comme la saison des pluies par exemple qui annihile toute fiabilité logistique pendant plus de deux mois dans la zone nord du Cameroun et sud du Tchad. Pour Cotonou, ce sont notamment les temps d'attente à l'ancre dus à la congestion chronique des terminaux et les délais considérables dans le traitement administratif et douanier des marchandises en transit qui obèrent la qualité et la fiabilité globale du corridor. Les deux solutions logistiques présentent aussi des contraintes de sûreté avec l'organisation couteuse et contraignante de convois sécurisés.

Chaque corridor présente ainsi une somme d'opportunités et de contraintes à mettre en relation avec chacun des marchés enclavés sélectionnés. Aussi, l'analyse ci-après combine les possibilités potentielles logistiques sur les 4 aires de marché enclavées et depuis les 4 corridors, ce qui signifie que 16 options de transport ont été appréhendées selon une méthodologie adaptée aux réalités de l'Afrique subsaharienne.

\section{POUR UN INDICATEUR DE PERFORMANCE LOGISTIQUE SIMPLIFIÉ ET ADAPTÉ}

Les auteurs ont adopté un double angle d'analyse. D'une part, il a été pris en considération les principaux index de performance logistique élaborés par les experts du secteur, en isolant particulièrement les outils méthodologiques constitués pour servir les analyses dans les zones en voie de développement. D'autre part, il a été intégré les constats et remarques issus des pratiques opérationnelles des logisticiens eux-mêmes exploitant des services sur un ou plusieurs corridors et desservant une ou plusieurs zones enclavées retenues dans le contexte de cette étude.

Sur le premier point, les travaux récents les plus intéressants recensent le Logistics Performance Index (LPI) de la Banque Mondiale ${ }^{12}$ auquel il peut être additionné les ajustements méthodologiques du Logistics Capability Index $(\text { LOCAI })^{13}$. De plus, des initiatives intéressantes 
issues du recensement des pratiques de transport les plus pénalisantes ont été considérées comme les travaux et rapports de l' «Observatoire des Pratiques Anormales » soutenu directement par le Conseil des Chargeurs Burkinabé. L'ensemble met en perspective l'impérieuse nécessité de considérer une somme importante de paramètres dans une vision quasi dynamique, permettant ainsi de considérer le poids de chacune des variables dans l'analyse de la performance logistique.

Toutefois, prenant en compte la complexité et la versatilité des conditions générales de transport et de marché de la vaste zone à l'étude, aucun indicateur idoine n'existe. Pour tenter de contrer ce problème et de pondérer chacune des variables dans une approche cohérente, un indicateur logistique a été créé de toutes pièces en prenant des "paramètres essentiels de calculs » qui considèrent autant des facteurs quantitatifs que qualitatifs. Ce travail conduit à des étalonnages logistiques qui traduisent, de facto un jugement avec des subjectivités issues des priorités, des opinions ou encore des objectifs fixés. Les étalonnages demeurent relatifs puisqu'ils font références à des comparaisons. Ici, l'objectif n'est pas de démontrer si un corridor est meilleur que l'autre. Il est d'ailleurs précisé à la section précédente qu'un corridor peut procurer différentes valeurs selon l'approche adoptée ou les objectifs fixés. Les conditions économiques, sociales ou environnementales qui ont une durée variable auront toujours un impact sur les résultats obtenus qui dépendent du moment précis où la collecte des données a été effectuée. De ce fait, l'étalonnage est avant tout un outil permettant de se positionner et de quantifier ou qualifier l'évolution temporelle ou les impacts d'un changement infrastructurel ou administratif. Un outil d'étalonnage procure aux acteurs d'un corridor un moyen de se comparer par rapport à une situation antérieure selon les meilleures informations dont ils disposent, tenant compte du fait que ces mêmes acteurs disposent souvent d'informations privilégiées, mais souvent confidentielles.

Selon certains auteurs ${ }^{14}$, les indicateurs utilisés dans le cadre de projets portant sur le commerce et le transport régional devraient offrir les caractéristiques suivantes : $1^{\circ}$ Etre faciles à mesurer et à colliger; $2^{\circ}$ Être basés sur les paramètres pertinents et compris; $3^{\circ}$ Permettre d'identifier des coûts ou des délais excessifs; $4^{\circ}$ Être, dans la mesure du possible, déjà colligés régulièrement par les principaux intervenants logistiques.

\section{L'inventaire des travaux effectués par les agences internationales et le milieu académique pour mesurer la performance du transport ou des corridors révèlent qu'il existe au-delà de 120 indicateurs potentiels.}

L'inventaire des travaux effectués par les agences internationales et le milieu académique pour mesurer la performance du transport ou des corridors révèlent qu'il existe au-delà de 120 indicateurs potentiels. En adoptant une perspective critique, plusieurs indicateurs s'avèrent difficilement applicables dans le contexte subsaharien du fait de l'indisponibilité des données. Ensuite, la production d'un certain nombre d'indicateurs reste tributaire de la réalisation d'enquêtes spécifiques ou sectorielles qui ne peuvent être réalisées sans avoir accès à des fonds et des appuis organisationnels conséquents. Enfin, la plupart des indicateurs produits par les organisations internationales relèvent de l'échelle nationale, trop souvent incompatible avec les problématiques transnationales d'un corridor de transport. Dans le cas présent, la solution adoptée consiste à construire un indicateur à partir de données nationales mais avec des ajustements qualitatifs comme, par exemple, l'analyse d'un corridor routier qui va cumuler des données routières sur tous les États traversés. Si l'on considère les indicateurs institutionnels, la valeur finale retenue aura préalablement subie une analyse pondérée en fonction de la distance parcourue dans chaque État traversé.

La présente matrice d'étalonnage utilise des données accessibles et publiques. Elle comporte trois volets d'analyse sur les plans opérationnel, institutionnel et commercial. D'abord, le volet opérationnel se consacre à l'évaluation de l'infrastructure de transport sur le corridor et à sa connectivité avec les marchés internationaux $(25 \%)$. Ensuite, le volet institutionnel et com- 
mercial vise à déterminer le niveau d'organisation du transit douanier et terrestre, la sûreté et la sécurité du transit et les mesures de facilitation du commerce (25\%). Enfin, le volet coût évalue l'accessibilité financière du corridor $(50 \%)$.

Le poids relatif de chaque indicateur $(25 / 25 / 50)$ est donné à titre indicatif, car c'est cette pondération qui a été retenue pour la présente analyse (Figure 4). Toutefois, au regard de la grande hétérogénéité des produits qui circulent sur les corridors, un utilisateur de l'indicateur pourrait très bien vouloir modifier la pondération pour apporter plus ou moins d'importance à un indicateur plutôt qu'à un autre. L'architecture de la matrice sur tableur permet une grande souplesse dans l'ajustement des paramètres selon les «commandes et contraintes » des différentes catégories d'utilisateurs. À titre d'exemple, la sécurisation de trafics à haute valeur ou la fiabilité logistique relative pour des trafics périssables peuvent être prises en compte avec des sur et sous pondérations pour une évaluation globale sur mesure de la performance.

Figure 3 : Détail des indicateurs opérationnels, institutionnels, commerciaux et tarifaires et leur pondération dans le total d'évaluation ${ }^{7}$

\begin{tabular}{|c|c|c|}
\hline Volet / Indicateur / Donnée (indicateur) & Source & Poids (\%) \\
\hline Opérationnel: & & 25 \\
\hline Durée du transit portuaire & & 7,5 \\
\hline Durée des procédures administratives et douanières & Banque mondiale (entreprisesurveys.org) & \\
\hline Connectivité internationale & & 2,5 \\
\hline Services de lignes & CNUCED / Liner shipping connectivity index & \\
\hline Durée du transit terrestre & & 10 \\
\hline Distance routière & Digital Chart of the World et cartes actualisées & \\
\hline Distance ferroviaire & Digital Chart of the World et cartes actualisées & \\
\hline Vitesses moyennes sur tronçons & Rapports sectoriels et blogues & \\
\hline État des réseaux terrestres & Rapports sectoriels et blogues & \\
\hline Temps de rupture de charge & Rapports sectoriels et données primaires de PSL & \\
\hline Passage de frontière & Rapports sectoriels et LPI & \\
\hline Qualité du réseau routier & & 2,5 \\
\hline Proportion du réseau asphalté & International Road Federation & \\
\hline Densité du réseau routier & International Road Federation & \\
\hline Technologies de l'information et des télécommunications & & 2,5 \\
\hline Couverture de la téléphonie mobile & Union internationale des télécommunications & \\
\hline Institutionnel et commercial : & & 25 \\
\hline Organisation du transit douanier & & 5 \\
\hline Efficience du transit douanier & LPI & \\
\hline Nombre de documents requis à l'import & Doing Business et LPI & \\
\hline Sûreté et sécurité & & 5 \\
\hline Morbidité routière par habitant & Organisation mondiale de la Santé & \\
\hline Distance routière & Digital Chart of the World & \\
\hline Fiabilité & & 15 \\
\hline Ponctualité des envois & LPI & \\
\hline Perception de la corruption & Transparency International & \\
\hline Compétence des PSL locaux & LPI & \\
\hline Distance routière & DCW & \\
\hline Coûts & & 50 \\
\hline Transit terrestre & Données primaires et estimations & 50 \\
\hline
\end{tabular}

Dans le but de garder les choses simples, le résultat de chaque indicateur s'exprime sur une échelle de 0 à 5 où 5 équivaut au meilleur résultat sur un corridor donné. La formule retenue pour rapporter les données sur cette échelle est la suivante: $5 \mathrm{x}$ (résultat $\mathrm{du}$ corridor/meilleur résultat).

Lorsque des résultats plus élevés correspondent à une moins bonne performance (par exemple les critères de distance ou de temps), la formule utilisée et présentée ci-dessous inverse l'opé- ration pour que le résultat le plus bas donne la meilleure note : 5 x (meilleur résultat/résultat du corridor)

Certains indicateurs utilisés comme source de référence tels que ceux provenant du LPI sont déjà exprimés sur une échelle de 1 à 5 . Pour les replacer dans leur contexte de concurrence régionale, la même formule a été appliquée et le minimum observé est la note octroyée par le LPI pour les pays concernés dans la zone à l'étude. 


\section{QUELQUES PREMIERS RÉSULTATS EN GUISE DE SYNTHÈSE : LE CAS DU CORRIDOR LIBYEN}

L’infrastructure projetée devient soudainement un outil pragmatique d'intégration continentale capable, entre autres, de fédérer les États et de soutenir le développement économique des parties les plus pauvres du continent.

En décembre 2008, la Banque africaine de Développement annonçait qu'elle allait étendre ses champs de partenariat avec la Libye, un pays qui promeut alors le caractère vital de la construction d'un tronçon routier reliant la frontière sud de la Libye à Agadès et Zinder au Niger. L'infrastructure projetée devient soudainement un outil pragmatique d'intégration continentale capable, entre autres, de fédérer les États et de soutenir le développement économique des parties les plus pauvres du continent. Les acteurs publics, privés et institutionnels de l'Afrique subsaharienne portent un vif intérêt à ce projet infrastructurel structurant d'envergure géographique et géostratégique unique.

L'indicateur de performance logistique, avec l'appui des institutions publiques Libyennes et d'opérateurs logistiques privés, a pu être activé avec un étalonnage qui permettait de calibrer le corridor libyen face aux corridors terrestres déjà en place pour desservir les zones enclavées. À la lumière des résultats présentés à la figure 5 , il convient d'admettre que la performance du corridor libyen demeure bien en deçà des corridors utilisés traditionnellement pour rejoindre le Niger et le Tchad. Le niveau de performance constaté est tributaire de deux principaux facteurs. D'une part, avec un poids de $50 \%$ pour le coût, le corridor traversant le Sahara se trouve nettement désavantagé notamment en raison des distances géographiques à couvrir. D'autre part, les fréquentations encore très faibles de l'actuel réseau joue en défaveur $\mathrm{du}$ corridor qui reste extrêmement coûteux par unité transportée.

Aujourd'hui encore, son utilisation est limitée à quelques ravitaillements humanitaires d'outremer ou provenant directement de la Libye. Malgré un réseau routier performant jusqu'aux frontières tchadiennes et nigériennes, les réseaux demeurent difficiles et risqués. En effet, les prestataires de services logistiques libyens ne sont disposés à assurer des services vers les régions sahéliennes qu'à des tarifs pouvant être qualifiés de prohibitifs pour certains produits. Il en coûte par exemple deux fois plus cher d'expédier un conteneur de 20 pieds de Misurata à Abéché que d'expédier le même conteneur depuis le port de Douala (Figure 4).

Malgré un réseau routier performant jusqu'aux frontières tchadiennes et nigériennes, les réseaux demeurent difficiles et risqués. En effet, les prestataires de services logistiques libyens ne sont disposés à assurer des services vers

les régions sahéliennes qu’à des tarifs pouvant être qualifiés de prohibitifs pour certains produits.

Figure 4 : Résultats synthétiques de la performance logistique globale sur les principaux corridors de transport desservant les marchés intérieurs Tchadiens et Nigériens ${ }^{7}$

\begin{tabular}{llcccc}
\hline Corridor & & $\begin{array}{c}\text { Opérationnel } \\
(25 \%)\end{array}$ & $\begin{array}{c}\text { Institutionnel et } \\
\text { commercial }(25 \%)\end{array}$ & $\begin{array}{c}\text { Coûts } \\
(50 \%)\end{array}$ & Total \\
\hline Douala & Abéché & 2,45 & 4,54 & 5,00 & 4,25 \\
Misurata & Abéché & 4,48 & 4,63 & 2,22 & 3,38 \\
Port Soudan & Abéché & 3,08 & 3,92 & 4,65 & 4,07 \\
\hline Cotonou & Agadès & 2,93 & 4,57 & 5,00 & 4,38 \\
Misurata & Agadès & 4,68 & 4,56 & 1,24 & 2,93 \\
\hline Douala & N'Djamena & 2,78 & 4,31 & 5,00 & 4,27 \\
Cotonou & N'Djamena & 2,49 & 4,24 & 3,91 & 3,64 \\
Misurata & N'Djamena & 4,66 & 4,00 & 1,54 & 2,94 \\
\hline Cotonou & Niamey & 3,24 & 4,57 & 5,00 & 4,45 \\
Misurata & Niamey & 4,68 & 4,43 & 0,66 & 2,61 \\
\hline
\end{tabular}


Sur le volet institutionnel et commercial, les données et indicateurs utilisés tendent à démontrer qu'il existe une certaine homogénéité d'un corridor à l'autre. Cela ne signifie pas nécessairement des qualités globales de services identiques mais les contraintes inhérentes à chaque corridor demeurent sensiblement les mêmes. Par contre, le volet opérationnel démontre qu'il existe des différences significatives entre chaque option de transport. Ces différences proviennent essentiellement de la durée des transits qui restent difficilement compressibles au regard de la qualité générale des infrastructures disponibles sur les tronçons routiers de la sous-région.

Tel qu'illustré à la figure 5, la performance du corridor libyen demeure supérieure à tous les autres, notamment grâce aux temps de transit portuaire et terrestre. La différence s'avère même intéressante en terme de positionnement concurrentiel sur Abéché en comparaison des circuits au départ de Douala ou de Port Soudan.

Figure 5 : Résultats détaillés des données composant le volet opérationnel de l’indicateur de performance logistique

\begin{tabular}{llccccc}
\hline Corridor & & $\begin{array}{c}\text { Durée port } \\
(30 \%)\end{array}$ & $\begin{array}{c}\text { Connectivité } \\
(10 \%)\end{array}$ & $\begin{array}{c}\text { Durée terre } \\
(40 \%)\end{array}$ & $\begin{array}{c}\text { Qualité du réseau } \\
(10 \%)\end{array}$ & TIC (10\%) \\
\hline Douala & Abéché & 1,25 & 5,00 & 2,20 & 2,95 & 3,99 \\
Misurata & Abéché & 5,00 & 2,42 & 5,00 & 3,73 & 3,63 \\
Port Soudan & Abéché & 0,81 & 2,89 & 4,33 & 3,10 & 5,00 \\
\hline Cotonou & Agadès & 0,91 & 5,00 & 3,61 & 2,15 & 5,00 \\
Misurata & Agadès & 5,00 & 2,25 & 5,00 & 5,00 & 4,55 \\
\hline Douala & N'Djamena & 1,25 & 4,65 & 2,95 & 2,75 & 4,83 \\
Cotonou & N'Djamena & 0,91 & 5,00 & 2,60 & 1,76 & 5,00 \\
Misurata & N'Djamena & 5,00 & 2,25 & 5,00 & 5,00 & 4,38 \\
\hline Cotonou & Niamey & 0,91 & 5,00 & 4,38 & 2,15 & 5,00 \\
Misurata & Niamey & 5,00 & 2,25 & 5,00 & 5,00 & 4,55 \\
\hline
\end{tabular}

Malgré une distance nettement supérieure à celle des autres corridors, les procédures douanières et portuaires accélérées en Libye permettent aux marchandises d'amorcer le transit terrestre bien plus tôt qu'ailleurs. Les délais pour libérer les marchandises du port de Misurata sont de 6 jours contre 24 jours pour Douala ou même 37 jours pour Port Soudan. Ces éléments de différenciation commerciale peuvent peser dans les choix stratégiques d'opération des corridors.

\section{Malgré une distance nettement supérieure à celle des autres corridors, les procédures douanières et portuaires accélérées en Libye permettent aux marchandises d'amorcer le transit terrestre bien plus tôt qu'ailleurs.}

L'option Libyenne demeure non-compétitive mais les distributions de résultats par volets (opération/institution-commerce/coût) démontrent les efforts à produire et peuvent se traduire en actions correctives. L'utilisation de l'indice de performance logistique soutient une approche qualitative globale et met en perspective les solutions d'investissements pour améliorer de manière ciblée les situations de transport rencontrées dans la sous-zone à l'étude. 


\section{CONCLUSION}

\section{Il ne faudrait pas moins de $\mathbf{2 0}$ milliards de dollars américains par an pour reconstituer, reconstruire et densifier les réseaux terrestres afin d'améliorer la fluidité des échanges intra-régionaux et internationaux.}

Selon l'ONU, il ne faudrait pas moins de 20 milliards de dollars américains par an pour reconstituer, reconstruire et densifier les réseaux terrestres afin d'améliorer la fluidité des échanges intra-régionaux et internationaux avec notamment un accent particulier sur la fiabilisation des accès directs aux principaux ports de la sous-région (Ouest-Afrique). En contrepoids, les mannes économiques et financières issues des exportations minières et énergétiques $\mathrm{du}$ continent africain pèsent plusieurs dizaines de milliards par an. Entre les deux, des infrastructures structurantes (routes, voies ferrées, quais et appontements portuaires) voient le jour plus pour satisfaire les besoins d'écoulement vers les marchés internationaux que pour soutenir le développement des économies nationales africaines. Il en résulte une situation logistique originale avec des réseaux qui se densifient au gré de l'intérêt particulier des grandes multinationales en fonction de la localisation géographique des matières premières exportables mais sans tenir compte des intérêts et besoins en infrastructures essentielles de transport.

Le calcul et l'utilisation d'un indice de performance logistique s'inscrit dans une forme de servitude aux intérêts massifiés d'entreprises internationales impliquées sur quelques corridors construits parfois de manière " artificielle » en fonction de leurs propres besoins d'extraction. Dans le cas de la présente étude, la simplification de l'indice développé permet de répondre aux multiples scénarii proposés dans le cadre de la desserte logistique des zones enclavées. Il permet de pondérer les éléments qualitatifs et quantitatifs dans une approche qui établit un lien entre approche méthodologique académique et résultats pragmatiques des données de terrain. La considération de facteurs logistiques « endogènes » dans le présent indice permet d'affiner la compréhension de la dynamique des réseaux de transport dans la stimulation économique régionale. Toutefois, il serait essentiel pour un indice de performance logistique de considérer des facteurs nonlogistiques « exogènes » comme l'amélioration sanitaire d'une zone enclavée par exemple. Il est admis que l'ouverture d'un tracé routier a d'importants impacts (positifs et négatifs) sur les populations et les environnements traversés par ces pénétrantes de transports. Il est admis aussi que l'ouverture d'une ligne ferroviaire peut changer la dynamique économique d'une région enclavée et soutenir une plus grande mobilité des populations. Toute une batterie d'éléments plus qualitatifs et sociétaux concernant directement le bien être des populations ou les incidences environnementales par exemple pourrait s'intégrer dans le calcul global de la performance logistique.

\section{Toute une batterie d'éléments plus qualitatifs et sociétaux concernant \\ directement le bien être des populations ou les incidences environnementales par exemple pourrait s'intégrer dans le calcul global de la performance logistique.}

Dans ce sens, il demeure probant qu'il faille encore très largement affiner les indicateurs de performance logistique dans le but d'intégrer toute la complexité des impacts positifs et négatifs des actions anthropiques visant à transporter des biens et des personnes depuis et vers les zones les plus reculées de la planète. Un champ académique, opératoire et appliqué reste à explorer.

\section{BIBLIOGRAPHIE}

${ }^{1}$ Debrie, J. (2001). " De la continentalité à l'Etat enclavé. Circulation et ouverture littorales des territoires intérieurs de l'ouest africain ». Thèse, Université du Havre, Centre interdisciplinaire de Recherches en Transports et Affaires internationales. ${ }^{2}$ Alix, Y. (2007). " Réflexions ouvertes sur les conditions $\mathrm{du}$ transit des marchandises en provenance et à destination des pays sans littoral». 
TRANSLOG / AFRICA. Séminaire International. Ouagadougou, Burkina Faso. 9 au 11 Octobre. Pedersen, P. O. (2001). «Freight transport under globalisation and its impact on Africa ». Journal of Transport Geography, 9, 85-99.

${ }^{3}$ Teravaninthorn, S.; Raballand, G., (2009). Transport Prices and Costs in Africa - A Review of the International Corridors. The International Bank for Reconstruction and Development / The World Bank. 145p.

${ }^{4} L$ 'ANTENNE, 13 juillet 2009, p3.

${ }^{5}$ Traore, A., (2007). «Les coûts de transport des marchandises des pays sans littoral, quelles solutions?». TRANSLOG / AFRICA. Séminaire International. Ouagadougou, Burkina Faso. 9 au 11 Octobre.

${ }^{6}$ Arvis, J.F. ; Raballand G. ; Marteau, J.F., (2007). « The Cost of being landlocked : Logistics Costs and Supply Chain Reliability ». Policy Research Working Paper $\mathrm{N}^{\circ}$ 4258. World Bank. 81p.

${ }^{7}$ Pelletier, J.F. Alix, Y. (2008). "Tools \& Methods for Improving the Analysis of the Inland Freight Trade Corridor in the Emerging Countries". 7th PanAfrican Port Conference. Djibouti, 15th to 18th of December.

${ }^{8}$ Nordas, H.K., (2006). « Time as a trade barrier: Implications for low-income countries». OECD Economic Studies, no. 42.

${ }^{9}$ Redding, S., Venables, A.J. (2002). « The Economics of Isolation and Distance » Nordic Journal of Political Economy vol. 28, pp. 93-108.

${ }^{10}$ Journal de la marine marchande, «Translog Africa (2007) : le défi maritime des pays enclavés »., $\mathrm{N}^{\circ}$ 4584-4585 - 9 Novembre 2007, 5-8.

${ }^{11}$ Pelletier, J.F., Alix, Y. (2009). "Benchmarking the Integration of African Corridors in International Value Chains" 2009 International Workshop 'Integrating Transport in Value Chains". June, $10^{\text {th }}$ to $12^{\text {th }}$, Montreal. Canada.

${ }^{12}$ Arvis, J-F., Mustra, M.A., Panzer, J., Ojala, L. and Naula, T., (2007). Connecting to Compete:Trade Logistics in the Global Economy; The Logistics Performance Index and Its Indicators, The World Bank.

${ }^{13}$ Memedovic, O, Ojala, L., Rodrigue, J.-P, Naula, T., (2008). Fueling the global value chains: what role for logistics capabilities? International Journal of Technological Learning, Innovation and Development 1(3), 353-374.

${ }^{14}$ Raballand, G., Marteau, J.-F., Kunka, C., Kabanguka, J.-K., Hartmann, O., (2008), Lessons of Corridor Performance Measurement, Sub-Saharan Africa Transport Policy Program, Discussion Paper $\mathrm{n}^{\circ} 7,21$ pages. 


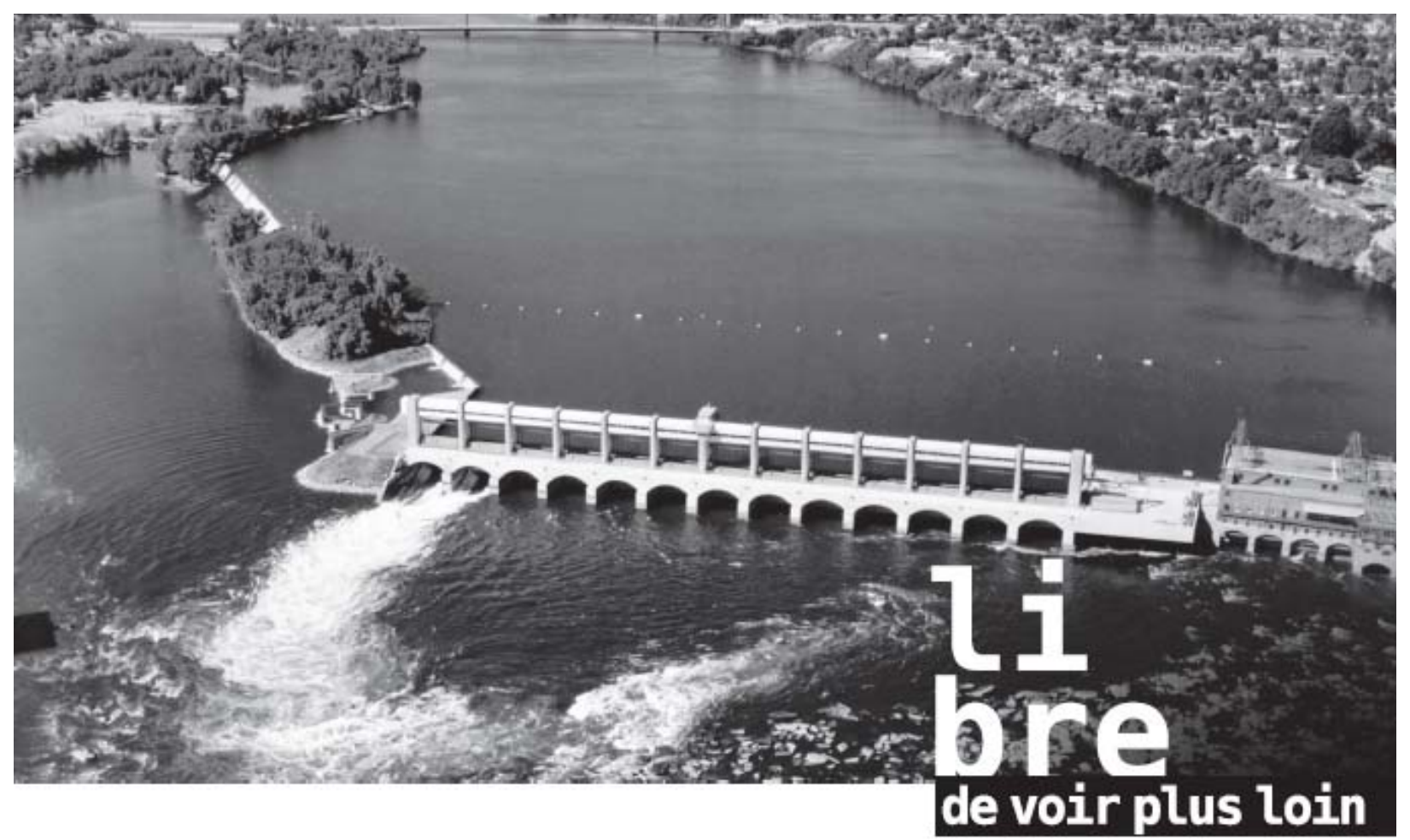

\section{DOCTORAT \\ EN MANAGEMENT DE PROJETS}

Dès septembre 2011

- Programme original, unique et novateur

- Possibilités de bourses de prestige et de soutien financier

- Programme limité à cinq admissions : faites vitel Date limite : 16 mai

Pour information :

Université du Québec à Chicoutimi 418 545-5011, poste 5282

uqac.ca/dmp 